\title{
RELIGIOSIDADE E ESPIRITUALIDADE EM ONCOLOGIA: CONCEPÇÕES DE PROFISSIONAIS DA SAÚDE'
}

\author{
Caroline Amado Gobatto \\ Tereza Cristina Cavalcanti Ferreira de Araujo
}

Resumo: A literatura especializada vem identificando influências positivas e negativas de crenças religiosas e espirituais no enfrentamento de enfermidades, tais como o câncer. Sendo assim, a presente investigação teve como objetivo conhecer, analisar e compreender as concepções de profissionais da saúde acerca da associação entre religiosidade, espiritualidade e saúde em Oncologia. Em uma primeira etapa, utilizou-se um questionário eletrônico, respondido por 85 profissionais. Na etapa posterior, sete profissionais participaram de grupos focais online. Os resultados indicaram que aproximadamente metade dos participantes da primeira etapa não praticam atividades religiosas. Porém, $85 \%$ revelaram grau elevado de espiritualidade. Temas religiosos/ espirituais são frequentes nos atendimentos. Os integrantes dos grupos relataram não ter recebido formação acadêmica para abordar a temática. Em suma, os participantes evidenciaram concepções positivas sobre a influência da religiosidade/ espiritualidade para a saúde, com efeitos negativos em menor proporção.Pesquisas futuras podem subsidiar programas destinados à capacitação dos futuros profissionais da área oncológica.

Palavras-chave: Religiosidade. Espiritualidade. Enfrentamento. Câncer. Profissionais da saúde.

1 Trabalho derivado da dissertação de mestrado da primeira autora, desenvolvida sob a orientação da segunda autora. Apoio financeiro: CNPq 


\section{Introdução}

Nas sociedades ocidentais, religião e ciência possuem um histórico de conflitos que se estende ao campo da saúde. Assim, desde a Revolução Científica, iniciada no século XVI, o enfoque explicativo das doenças supervalorizou características físico-biológicas e buscou excluir aspectos psíquicos, sociais e espirituais, contribuindo para a supremacia do paradigma biomédico. Porém, no decorrer do século XX, diversas transformações foram suscitadas pelo acompanhamento prolongado de enfermos crônicos e a constatação da influência de fatores psicológicos para a saúde (Castañon, 2007; Straub, 2005). Progressivamente, em saúde, reafirma-se o paradigma biopsicossocial, que preconiza a atenção integral pela inclusão de parâmetros políticos, econômicos e culturais (Gurung, 2010; Straub, 2005).

Cabe lembrar que até mesmo a Organização Mundial da Saúde passou a destacar a dimensão espiritual em sua delimitação conceitual da saúde, propiciando a ampliação do escopo das pesquisas na área (Organização Mundial da Saúde, 1999). De fato, é notável o aumento das publicações que almejam relacionar saúde e religiosidade/espiritualidade. Contudo, no Brasil, a produção encontra-se muito aquém das publicações internacionais em termos quantitativos. Mas, um exame atento dessas produções, além de revelar um interesse crescente, indica também que os autores têm uma visão positiva da influência de aspectos religiosos e espirituais para a saúde dos indivíduos (Lucchetti, Almeida, \& Granero, 2010; Liberato \& Macieira, 2008; Moreira-Almeida, 2010; Paiva et al., 2009; Panzini \& Bandeira, 2007).

\section{Enfrentamento religioso/espiritual}

Inicialmente, é preciso realçar a essência multidimensional das noções de religião, religiosidade, espiritualidade, crenças e fé, não viabilizando, portanto, definições consensuais (Dalgalarrondo, 2008).

James (1902/1994) focaliza a vivência pessoal da religião, definida como experiências, atitudes e sentimentos dos homens em sua relação individual com o divino. De maneira distinta, Geertz (1973/2008) sublinha a dimensão cultural e simbólica da religião, destacando o papel dos símbolos sagrados na configuração da visão de mundo de cada indivíduo.

Para alguns estudiosos, a religião engloba características comportamentais, sociais e doutrinárias específicas compartilhadas por um grupo e praticadas pelo indivíduo; ao passo que a espiritualidade está relacionada ao transcendente e ao direcionamento de questões sobre 
o significado da vida não necessariamente presentes em crenças e práticas religiosas (Fetzer Institute, 1999; Rocha, Panzini, Pargendler, \& Fleck, 2008; Sulmasy, 2009). Ademais, uma certa dicotomia entre religiosidade e espiritualidade pode ser apreendida em trabalhos que as distinguem respectivamente em: institucionalização e individualidade; estagnação e dinamismo; objetividade e subjetividade; crenças e experiências/emoções; negativa e positiva (e. g. Zinnbauer \& Pargament, 2004). Todavia, Pargament (1997) alerta que ambas estão intimamente conectadas, não se justificando a pressuposição de uma "espiritualidade-pessoal" sempre positiva e de uma "religiosidade-institucional" sistematicamente prejudicial. Segundo esse autor, há inúmeros exemplos empíricos que contradizem esses pré-julgamentos, a despeito de existir no meio acadêmico a preferência de alguns estudiosos pela espiritualidade ao invés da religião.

Em geral, denomina-se como enfrentamento o conjunto de esforços cognitivos e comportamentais destinados ao manejo de situações externas e/ou demandas internas que excedem os recursos pessoais do indivíduo e ocasionam sua sobrecarrega (Folkman, Lazarus, Gruen, \& DeLongis, 1986). Especificamente, quando o indivíduo utiliza a religião ou a fé como estratégia de manejo do estresse, a literatura reconhece o enfrentamento religioso/espiritual (ERE), cujas estratégias podem ser classificadas como positivas ou negativas, em função das consequências para a saúde dos indivíduos. Como exemplo de ERE positivo, costuma-se mencionar: sentimentos de segurança e conforto, conexão com os outros e crença no sentido da vida. Em contraposição, o ERE negativo parece se associar a uma visão pessimista do mundo e pouca autoconfiança (Panzini \& Bandeira, 2007; Pargament, 1997; Pargament, Smith, Koenig, \& Perez, 1998; Tix \& Frasier, 1998).

Com relação à avaliação do ERE, destacam-se dois instrumentos: a RCOPE scale, desenvolvida por Pargament, Koenig e Perez (2000), e sua versão abreviada, BriefCOPE scale (Pargament, Tarakeshwar, Ellison, \& Wulff, 2001). No âmbito brasileiro, a adaptação e a validação do primeiro instrumento - nomeado como Escala de Coping Religioso-Espiritual (Escala CRE) - foi empreendida por Panzini (2004); já a versão abreviada foi adaptada e validada em uma amostra de indivíduos soropositivos e intitulada Escala Breve de Enfrentamento Religioso (Faria, 2004).

Modelos explicativos têm sido elaborados na tentativa de compreender a relação entre religiosidade/espiritualidade e saúde, englobando as dimensões comportamental (dietas, comportamento de risco), social (suporte emocional de membros da instituição religiosa), psicológica (crenças e cognições que influem no enfrentamento e na qualidade de vida) e fisiológica (práticas que afetam a resposta de estresse) (Fitchett \& Canada, 2010). 


\section{Religiosidade e espiritualidade no contexto oncológico}

Apesar dos avanços conquistados em Oncologia o câncer ainda desencadeia ideias de morte e finitude, somadas às vivências de restrições corporais, dores e sofrimentos, que geram questionamentos dos valores e do projeto existencial. Nesses momentos de ressignificação, religiosidade e espiritualidade podem ter efeito positivo para o paciente, por um lado, contribuindo para a diminuição das experiências negativas provocadas pelo câncer e, por outro lado, aprimorando sua qualidade de vida (Aquino \& Zago, 2007; Prada, 2006; Tarakeshwar et al., 2006; Thuné-Boyle et al., 2011).

Estudos com mulheres brasileiras apontaram correlações positivas entre apoio social e espiritualidade com o enfrentamento saudável do câncer de mama (Hoffmann, Müller, \& Frasson, 2006; Hoffmann, Müller, \& Rubin, 2006). De modo semelhante, a fé foi avaliada como fonte de conforto e segurança para pacientes com câncer cérvico-uterino (Oliveira, Fernandes, \& Galvão, 2005). Em pacientes laringectomizados, a religião foi considerada um suporte significativo (Aquino \& Zago, 2007). Pacientes idosos relacionaram a fé religiosa à esperança, ao equilíbrio e ao fortalecimento (Teixeira \& Lefèvre, 2008). Porém, experiências negativas como a manutenção da rigidez e de sentimentos de auto-condenação, também foram identificadas (Barbosa \& Freitas, 2009).

Diante da constatação de influências tanto positivas como negativas da religiosidade/espiritualidade, é incontornável que profissionais de saúde estejam atentos a tais dimensões, inclusive solicitando inclusive a colaboração de representantes religiosos ou de profissionais especializados nestas questões para o manejo clínico de seus pacientes oncológicos. Em escolas médicas americanas, disciplinas preparatórias para lidar com as questões religiosas e espirituais dos pacientes são comuns (Koenig, Hooten, Lindsay-Calkins, \& Meador, 2010). É preciso explicitar também que em muitos países da América do Norte e da Europa a figura do capelão está consolidada nas instituições hospitalares (Fitchett, King, \& Vandenhoeck, 2010). Ademais, segundo Maciejewski et al. (2012) a articulação com profissionais religiosos é essencial no acompanhamento de pacientes oncológicos e, especialmente, daqueles que se encontram em cuidados paliativos.

No Brasil, a legislação vigente estabelece diretrizes para serviços dessa natureza (Constituição Federal de 1988; Lei no 9.982/2000; Decreto № 30.582/2009), porém, ainda são escassas as iniciativas nesta direção. Aliás, em um estudo com médicos oncologistas, Junqueira (2008) verificou que, apesar dos esforços para contemplar a subjetividade dos profissionais em unidades de cuidados paliativos, impera o despreparo para lidar com a dimensão religiosa nos atendimentos. 
Tendo em vista a diversidade religiosa brasileira, bem como a sua elevada importância atribuída pela população, os estudantes das profissões de saúde necessitariam receber alguma formação sobre o assunto (Dal-Farra \& Geremia, 2010; Moreira-Almeida et al., 2010). A falta de treinamento e de habilidade em identificar as demandas dos usuários, assim como o receio de influenciar as crenças dos pacientes, constituem barreiras percebidas pelos próprios profissionais, que dificultam a abordagem da religiosidade/espiritualidade nos atendimentos (Balboni et al., 2007; McCauley et al., 2005; Monroe et al., 2003).

Em contraponto, diversos estudiosos ressaltam benefícios da religiosidade/espiritualidade para o enfrentamento de doenças, justificando a inclusão da temática nas intervenções em saúde (Balboni et al., 2007; McCauley et al., 2005; Monroe et al., 2003; Panzini \& Bandeira, 2007; Saad, Masiero, \& Battistella, 2001). Para alguns autores, isto propicia a compreensão das crenças dos pacientes e sua relação com a doença, permitindo detectar interferências negativas na adesão aos tratamentos (Koenig, 2006; Post, Puchalski, \& Larson, 2000).

O preparo dos profissionais da saúde brasileiros para lidar com a temática religiosa/espiritual também tem sido examinado (Peixoto, 2006; Tomasso, Beltrame, \& Lucchetti, 2011). Alves (2004) averiguou a opinião de jovens universitários de diversos cursos sobre o ensino religioso em disciplinas da graduação. Constatou-se a necessidade dos participantes em romper com as antigas tradições religiosas em prol de uma vivência da espiritualidade singular. Quanto às práticas de ensino, sugeriram-se debate crítico e livre expressão da opinião dos alunos sobre as doutrinas religiosas.

A religiosidade/espiritualidade dos profissionais da saúde também foram foco de estudos (Espíndula, Valle, \& Bello, 2010; Junqueira, 2008). Ecklund, Cadge, Gage e Catlin (2007) levantaram as crenças religiosas e espirituais de 74 oncopediatras. Do total, 22 respondentes $(29,7 \%)$ não participavam de cultos religiosos no último ano do curso, porém, 18 $(24,3 \%)$ realizaram tais atividades de duas a três vezes por mês. Mais da metade $(52,7 \%)$ presumiram que suas crenças podiam influenciar a interação com colegas e pacientes.

Tendo em vista tais evidências destacadas pela literatura especializada, realizou-se uma pesquisa com o objetivo geral de conhecer, analisar e compreender as concepções de profissionais da área da Oncologia sobre religiosidade, espiritualidade e saúde, bem como a abordagem dessa temática com pacientes em seus atendimentos. 


\section{Método}

Delineou-se um estudo descritivo e exploratório, quanti-qualitativo, organizado em duas etapas e previamente autorizado pelo Comitê de Ética em Pesquisa da Secretaria de Saúde do Distrito Federal. A primeira etapa foi conduzida com uma amostra de conveniência constituída por 85 profissionais com formação completa na área da saúde que atuavam na assistência a pacientes oncológicos. Desses, sete profissionais foram distribuídos em dois grupos focais online $(n=3$ e $n=4)$ na segunda etapa do estudo.

A pesquisa foi divulgada na rede de contatos profissionais da área de saúde, utilizando-se a estratégia comumente denominada "bola de neve". Inicialmente, a coleta de dados foi feita por meio de um questionário eletrônico elaborado pela ferramenta Survey Monkey e disponibilizado na internet. O conteúdo englobava: a) Termo de Consentimento Livre e Esclarecido (TCLE); b) perguntas destinadas ao levantamento de dados sociodemográficos, hábitos religiosos, crenças dos participantes e presença de aspectos religiosos/espirituais em seus atendimentos; c) Escala Breve de Enfrentamento Religioso (Faria, 2004), composta por 14 itens para avaliação de padrões positivo e negativo, medidos em uma escala tipo Likert (1-4); d) convite para participar da segunda etapa da pesquisa. Durante os grupos focais online, realizados em data e horário previamente agendados, adotaram-se TCLE específico e roteiro com cinco questões norteadoras da discussão, que também ocorreu em ambiente virtual, utilizando-se o software GVO Conference.

Os dados provenientes do questionário eletrônico foram submetidos à análise estatística descritiva (frequência e porcentagem) e inferencial (Teste $t$ ), com o auxílio dos programas Statistical Package for Social Science (SPSS versão 18.0) e do Excel Starter 2010. Os relatos reunidos ao longo dos grupos online foram submetidos à análise de conteúdo temática (Bardin, 2009).

\section{Resultados e discussão}

\section{Etapa questionário eletrônico}

A Tabela 1 apresenta os dados sociodemográficos relativos a amostra desta etapa. 
Tabela 1

Caracterização sociodemográfica dos participantes $(N=85)$

\begin{tabular}{|c|c|c|c|c|c|c|c|c|}
\hline \multirow{2}{*}{ Variável } & $\begin{array}{l}\text { Médico } \\
(n=17)\end{array}$ & $\begin{array}{l}\text { Nutricionista } \\
(n=16)\end{array}$ & $\begin{array}{c}\text { Psicólogo } \\
(n=40)\end{array}$ & \multicolumn{5}{|c|}{ Outros $^{\mathrm{a}} \quad(n=12)$} \\
\hline & $n$ & (\%) & $n$ & (\%) & $n$ & (\%) & $n$ & (\%) \\
\hline \multicolumn{9}{|c|}{ Gênero } \\
\hline Feminino & 14 & $(82,4)$ & 15 & $(93,7)$ & 33 & $(82,5)$ & 10 & $(83,3)$ \\
\hline Masculino & 3 & $(17,6)$ & 1 & $(6,3)$ & 7 & $(17,5)$ & 2 & $(16,7)$ \\
\hline \multicolumn{9}{|c|}{ Situação Conjugal } \\
\hline Com companheiro & 10 & $(58,8)$ & 8 & (50) & 14 & (35) & $7^{b}$ & $(58,3)$ \\
\hline Sem companheiro & 7 & $(41,2)$ & 8 & (50) & 26 & (65) & $4^{b}$ & $(33,3)$ \\
\hline \multicolumn{9}{|c|}{ Escolaridade } \\
\hline Superior & 2 & $(11,9)$ & 1 & $(6,3)$ & 7 & $(17,5)$ & 2 & $(16,7)$ \\
\hline Especialização & 9 & $(52,9)$ & 9 & $(56,3)$ & 13 & $(32,5)$ & 7 & $(58,3)$ \\
\hline Mestrado & 3 & $(17,6)$ & 3 & $(18,7)$ & 14 & (35) & 1 & $(8,3)$ \\
\hline Doutorado & 3 & $(17,6)$ & 3 & $(18,7)$ & 6 & (15) & 2 & $(16,7)$ \\
\hline \multicolumn{9}{|c|}{ Religião } \\
\hline Catolicismo & 7 & $(41,2)$ & $7^{c}$ & $(43,8)$ & 15 & $(37,5)$ & 4 & $(33,3)$ \\
\hline $\begin{array}{l}\text { Espiritismo } \\
\text { Kardecista }\end{array}$ & 2 & $(11,8)$ & $3^{c}$ & $(18,8)$ & 6 & (15) & 5 & $(41,7)$ \\
\hline Sem religião & 5 & $(29,4)$ & $2^{c}$ & $(12,5)$ & 10 & $(25)$ & 2 & $(16,7)$ \\
\hline Outros & 3 & $(17,6)$ & $3^{c}$ & $(12,5)$ & 9 & $(17,5)$ & 1 & $(8,3)$ \\
\hline \multicolumn{9}{|c|}{ Renda (salários mínimos) } \\
\hline Até 3 & 2 & $(11,8)$ & 1 & $(6,2)$ & 6 & $(15)$ & 1 & $(8,3)$ \\
\hline Entre 3 e 6 & 2 & $(11,8)$ & 7 & $(43,8)$ & 11 & $(27,5)$ & 4 & $(33,3)$ \\
\hline Entre 6 e 10 & 3 & $(17,6)$ & 2 & $(12,5)$ & 9 & $(22,5)$ & 2 & $(16,7)$ \\
\hline Acima de 10 & 10 & $(58,8)$ & 6 & $(37,5)$ & 14 & (35) & 5 & $(41,7)$ \\
\hline
\end{tabular}

Nota. a Outros profissionais: Assistente Social $(n=1)$; Assistente Social/Psicólogo Comportamental ( $n$ =1); Biólogo ( $n=1)$; Enfermeiros $(n=2)$; Fisioterapeutas $(n=3)$; Fonoaudiólogos $(n=2)$; Odontólogo $(n=1)$; Psico-Oncologista $(n=1)$; Técnico de enfermagem $(n=1)$.

${ }^{\mathrm{b}} n=11$

c $n=15$

As Figuras 1 e 2 caracterizam os hábitos religiosos dos participantes. Aproximadamente metade dos respondentes assinalou não participar de atividades religiosas públicas (instituições religiosas) ou privadas (leituras religiosas), denotando que tais atividades não fazem parte da rotina dos profissionais. Contudo, ao se comparar a frequência a institui- 
ções religiosas dos psicólogos da amostra, verificaram-se porcentagens próximas de profissionais que negaram (45\%) e aqueles que se consideraram muito assíduos (40\%). Sendo assim, verifica-se que os participantes dessa categoria profissional expuseram hábitos religiosos diversos.

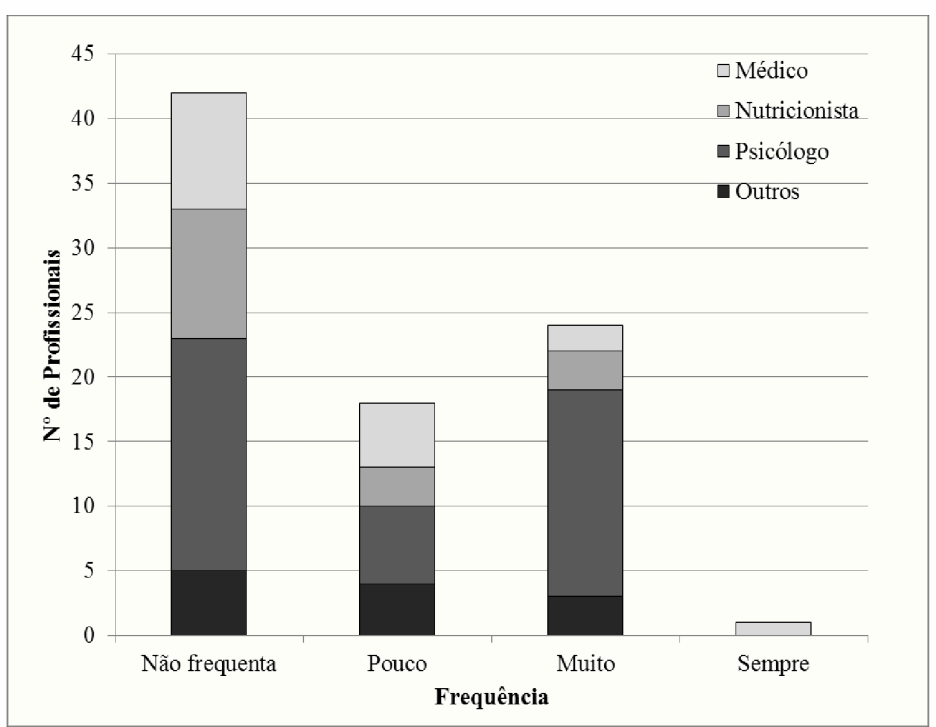

Figura 1. Grau de envolvimento em práticas religiosas públicas $(N=85)$.

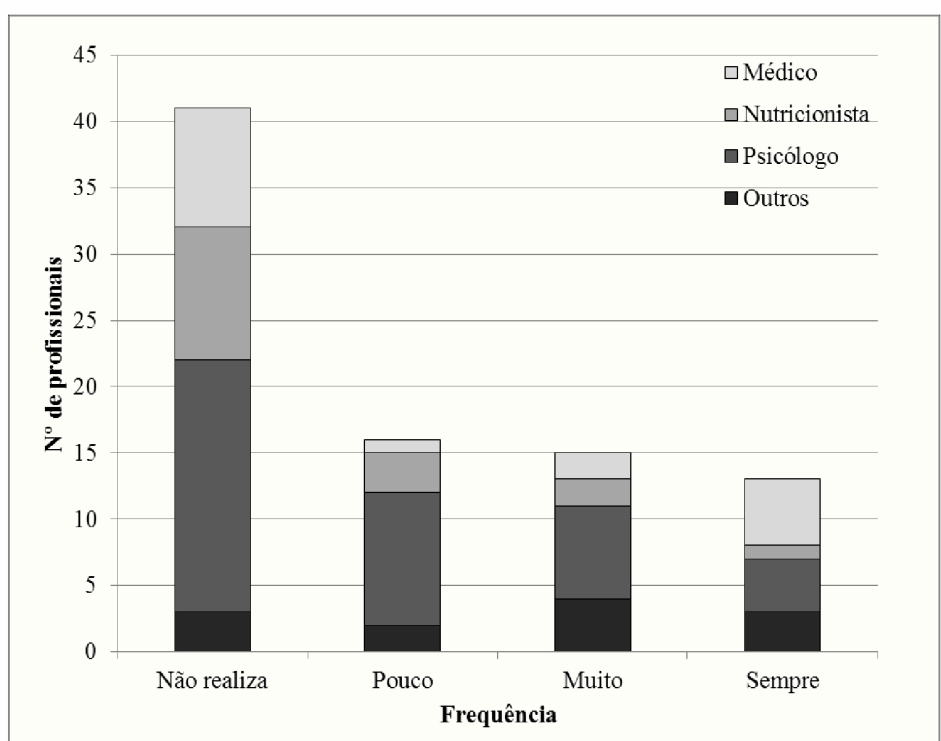

Figura 2. Grau de envolvimento em práticas religiosas privadas $(N=85)$. 
A maioria dos respondentes $(57,6 \%)$ se avaliou como muito ou completamente espiritualizada. Apenas dois médicos e um psicólogo negaram que a espiritualidade faça parte de suas vidas. Esse dado corrobora o estudo de Paiva (2002), cujos profissionais não se mostraram contrários à ideia de divindade desde que não fosse vinculada ao deus cristão. Tal como já discutido na literatura, é possível supor que os profissionais expressam necessidade de rompimento com as antigas tradições religiosas, aspirando vivenciar a espiritualidade de maneira singular (Alves, 2004).

A análise da percepção de médicos, nutricionistas e psicólogos sobre as influências que a religião pode exercer na saúde das pessoas mostrou maiores efeitos positivos do que negativos (Tabela 2). A exemplo de evidências já reportadas por vários pesquisadores, o benefício mais frequentemente assinalado foi o enfrentamento de doenças (Faria \& Seidl; 2005; Panzini \& Bandeira, 2007; Pargament, 1997; Pargament, 1998; Pargament et al., 2000; Pargament et al. 2001).

Tabela 2

Percepção sobre as influências da religiosidade/espiritualidade na saúde, segundo médicos, nutricionistas e psicólogos

\begin{tabular}{|c|c|c|c|c|c|c|c|}
\hline PROFISSIONAL & VARIÁVEL & $N$ & M & $D P$ & $G L$ & $T$ & $R$ \\
\hline \multirow{2}{*}{ MÉDICO } & IPS & 17 & 2,80 & 0,54 & \multirow{2}{*}{16} & \multirow{2}{*}{$7,37^{* * *}$} & \multirow{2}{*}{0,88} \\
\hline & INS & 17 & 1,50 & 0,53 & & & \\
\hline \multirow{2}{*}{ NUTRICIONISTA } & IPS & 16 & 2,75 & 0,48 & \multirow{2}{*}{15} & \multirow{2}{*}{$7,45^{* * *}$} & \multirow{2}{*}{0,89} \\
\hline & INS & 16 & 1,53 & 0,46 & & & \\
\hline \multirow{2}{*}{ PSICÓLOGO } & IPS & 39 & 2,77 & 0,51 & \multirow{2}{*}{38} & \multirow{2}{*}{$8,76^{* * *}$} & \multirow{2}{*}{0,82} \\
\hline & INS & 39 & 1,70 & 0,48 & & & \\
\hline
\end{tabular}

Nota. IPS = Influência Positiva na Saúde; INS: Influência Negativa na Saúde.

$n=$ número de respostas ao item; $M=$ média; $D P=$ desvio padrão; $\mathrm{GL}=$ graus de liberdade; $t=$ índice do Teste $t$ de Student; $r=$ tamanho do efeito ${ }^{* * *} p<0,001$

No que tange à solicitação de ajuda religiosa ou espiritual pelos pacientes oncológicos, pouco mais da metade dos participantes $(54,1 \%)$ respondeu afirmativamente, sendo que 23 eram psicólogos. Para $85 \%$ dos profissionais, os temas religiosos/espirituais são frequentes nos atendimentos. Quanto à abordagem da temática pelos profissionais da saúde, a maioria dos respondentes (52,9\%) informou que se deve lidar apenas 
com o que o paciente relatar. Ressalta-se que apenas nove respondentes expuseram que nenhum profissional da saúde deve abordar a temática nos atendimentos (Figura 3). Muitos estudiosos admitem que as vivências relacionadas à religião/espiritualidade do paciente sejam averiguadas atribuindo um papel ativo ao profissional da saúde no levantamento dessas informações desde que se assegure o respeito às crenças dos pacientes (Koenig, 2006; Panzini \& Bandeira, 2007; Saad et al., 2001).

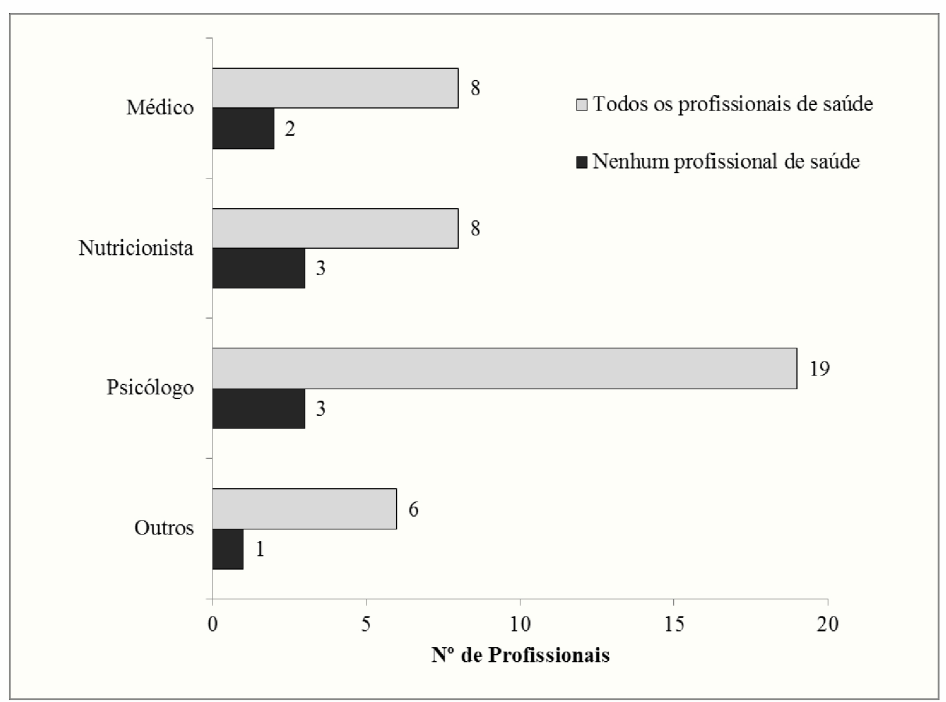

Figura 3. Percepção sobre a abordagem da temática religiosa/espiritual com pacientes oncológicos.

Quanto ao direcionamento das necessidades religiosas e espirituais dos pacientes oncológicos, aproximadamente metade dos participantes $(48,2 \%)$ concordou que não existe profissional responsável por esse tipo de apoio em seu local de trabalho. $O$ apoio do capelão foi mencionado por 15 participantes, o que representa menos de um quinto da amostra. Este resultado confirma que não há uma participação efetiva deste profissional em equipes de saúde, apesar da legislação reguladora que prevê a atuação dos capelães em instituições hospitalares brasileiras (Constituição, 1988; Lei no 9.982/2000; Decreto № 30.582/2009; Gentil, Guia, \& Sanna, 2011).

Embora a temática religiosa seja frequente nos atendimentos, a maioria dos profissionais do estudo não encaminhou pacientes a representantes religiosos na própria instituição $(83,5 \%)$. Esse dado pode estar relacionado ao fato de não haver um profissional de referência. $\mathrm{Na}$ implementação de um serviço de suporte religioso, estudiosos indicam ser necessário priorizar ações integradas entre o trabalho de profissionais da 
saúde e religiosos, respeitando-se as demandas da família e do paciente (Fitchett et al., 2010; Junqueira, 2008; Maciejewski et al., 2012).

A percepção dos profissionais da saúde sobre a influência da religiosidade/ espiritualidade na experiência oncológica é ilustrada na Figura 4. A questão "fonte de conforto" foi a mais selecionada pelos participantes, correspondendo a $97,6 \%$ da amostra. O item "pessimismo sobre a doença e o prognóstico" recebeu a menor quantidade de indicações. Em termos gerais, foram apontadas mais influências positivas do que negativas, tal como salienta a literatura (Faria \& Seidl; 2005; Fitchett \& Canada, 2010; Koenig, 2009; Panzini \& Bandeira, 2007; Pargament, 1997). É válido destacar, porém, que grande parte dos profissionais considerou que os pacientes podem atribuir ao câncer um caráter punitivo. Nesse sentido, estudos vêm alertando que a percepção da doença como punição está geralmente associada a maneiras desadaptativas do enfermo ao lidar com o câncer. Assim, o enfrentamento negativo deve ser averiguado pelo profissional em seus atendimentos a fim de que possam ser promovidas maneiras mais assertivas de lidar com a enfermidade (Barbosa \& Freitas, 2009; Maciejewski et al., 2012; Thuné-Boyle, Stygall, Keshtgar, Davidson, \& Newman, 2011).

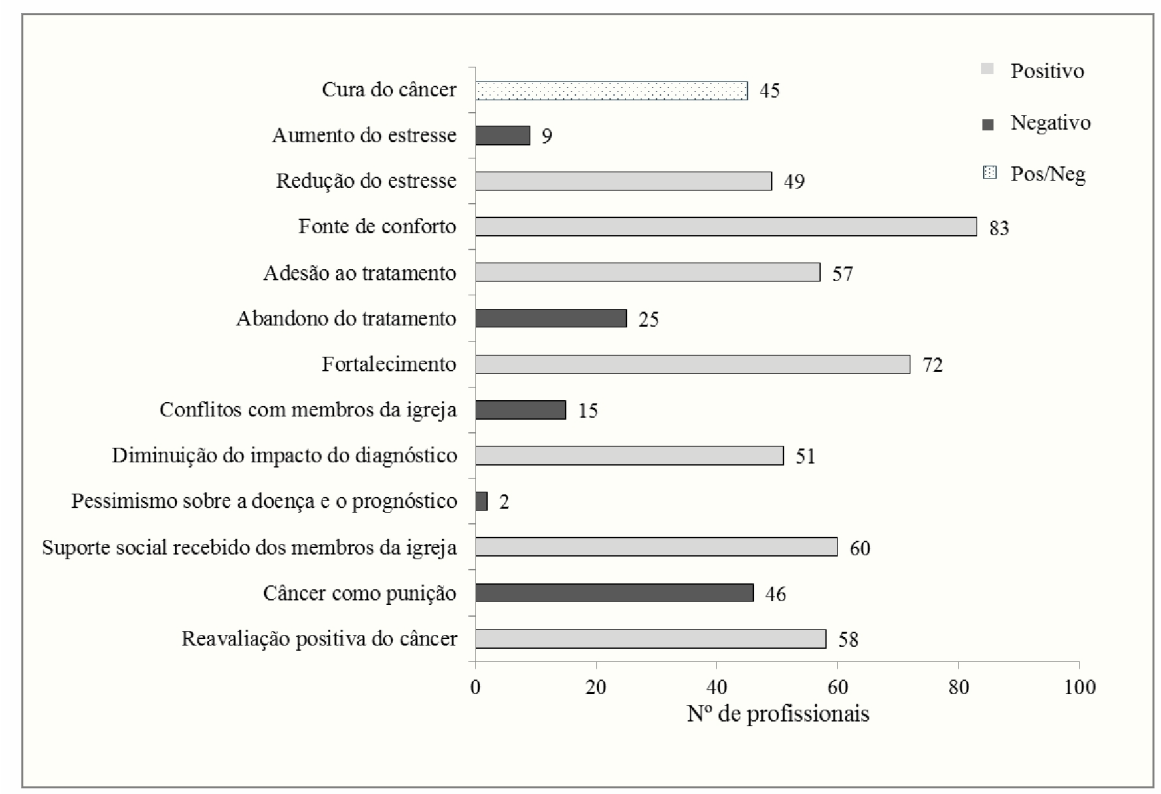

Figura 4.Percepção sobre as influências da religiosidade/espiritualidade na experiência oncológica do paciente.

A Escala Breve de Enfrentamento Religioso foi respondida por 82 participantes. Desses, 10,5\% sinalizaram não recorrer a estratégias religiosas/espirituais para lidar com situações de estresse. Mais da metade 
dos respondentes $(53,6 \%)$ indicou não utilizar estratégias de cunho negativo. Constatou-se, portanto, maior utilização de estratégias positivas em detrimento das estratégias negativas (Tabela 3).

Tabela 3

Comparação entre as médias de ERE positivo e ERE negativo de médicos, nutricionistas e psicólogos.

\begin{tabular}{|c|c|c|c|c|c|c|c|c|}
\hline PROFISSIONAL & ERE & $N$ & $M$ & $D P$ & $G L$ & $T$ & $P$ & $R$ \\
\hline \multirow{2}{*}{ MÉDICO } & POSITIVO & 17 & 2,40 & 1,11 & \multirow{2}{*}{16} & \multirow{2}{*}{5,07} & \multirow{2}{*}{0,000} & \multirow{2}{*}{0,78} \\
\hline & NEGATIVO & 17 & 1,07 & 0,14 & & & & \\
\hline \multirow{2}{*}{ NUTRICIONISTA } & POSITIVO & 16 & 2,59 & 1,00 & \multirow{2}{*}{15} & \multirow{2}{*}{4,82} & \multirow{2}{*}{0,000} & \multirow{2}{*}{0,78} \\
\hline & NEGATIVO & 16 & 1,23 & 0,33 & & & & \\
\hline \multirow{2}{*}{ PSICÓLOGO } & POSITIVO & 38 & 2,50 & 0,83 & \multirow{2}{*}{37} & \multirow{2}{*}{9,68} & \multirow{2}{*}{0,000} & \multirow{2}{*}{0,85} \\
\hline & NEGATIVO & 38 & 1,22 & 0,34 & & & & \\
\hline
\end{tabular}

Nota. $n=$ número de respostas ao item; $M=$ média; $D P=$ desvio padrão; $G \mathrm{~L}=$ graus de liberdade; $t=$ índice do Teste $t$ de Student; $p=$ significância; $r=$ tamanho do efeito

Os dados referentes ao ERE seguiram o padrão descrito pela literatura, ou seja, ao se comparar as estratégias, predominaram aquelas de influência positiva (Koenig 2009; Panzini \& Bandeira, 2007; Pargament, 1997). De acordo com Junqueira (2008), médicos oncologistas se apoiam nas próprias estratégias religiosas para o acompanhamento de pacientes que se encontram fora de possibilidades terapêuticas.

Vale ressaltar que as crenças religiosas e/ou espirituais dos profissionais podem influenciar sua interação com colegas e pacientes, bem como na construção de sua visão sobre a morte, tendo reflexos para a assistência e para a ética nos cuidados (Ecklund et al., 2007; Peixoto, 2006).

\section{Etapa grupo focal online}

O primeiro grupo foi composto por uma nutricionista (N1) e dois psicólogos (P1 e P2), ao passo que o outro grupo contou com um médico (M1), uma nutricionista (N2) e duas psicólogas (P3 e P4). Ambos tiveram representantes das Regiões Centro-Oeste, Nordeste e Sudeste. A média de idade do primeiro grupo foi 39,3 anos e 33 anos do segundo. Do total, quatro participantes declararam afiliação religiosa (dois católicos e dois espíritas) e três se intitularam sem religião. $O$ tempo médio de atuação 
foi de 13,3 anos no primeiro grupo e sete anos no segundo grupo. Com relação ao tempo de atuação em Oncologia, as médias foram similares ( $M$ $=4,8$ e $M=5$, respectivamente).

No que se refere à percepção dos profissionais sobre a influência da religiosidade/espiritualidade para experiência oncológica dos pacientes e de seus familiares/cuidadores, os participantes comentaram:

Acho que a religiosidade e a espiritualidade afetam o emocional que por sua vez influencia a saúde física, e isso tanto de forma positiva quanto de forma negativa, depende...(P4)

... conforto e maior facilidade de enfrentamento de situações limites... (M1)

...em geral tende a ser um tipo de crença muito central na vida das pessoas quando adoecem, pois oferece uma alternativa de reflexão existencial. (P1)

As famílias e pacientes tendem a sentirem abandonados por Deus.... e ficam um tanto perdidos até encontrarem uma saída para esse conflito. (P1)

De modo geral, foram relatadas influências predominantemente positivas da religiosidade/espiritualidade tanto para os pacientes como para os familiares, o que está de acordo com estudos de autores brasileiros e de outros países (Aquino \& Zago, 2007; Barbosa \& Freitas, 2009; Fitchett \& Canada, 2010;Thuné-Boyle et al., 2011). Entretanto, a influência negativa da espiritualidade/religiosidade também foi destacada pelos integrantes dos grupos, assim como registrado na literatura (Barbosa \& Freitas; Fitchett \& Canada, 2010; Tarakeshwar et al., 2006; Thuné-Boyle et al., 2011).

É relativamente comum que os pacientes recorram à religião para lidar com o sofrimento, especialmente nos períodos de crises que intensificam as inúmeras dificuldades experienciadas (Dalgalarrondo, 2008; Geertz, 1973/2008). Assim, do ponto de vista dos participantes, as explicações religiosas/espirituais podem propiciar tanto conforto e reavaliação positiva como vincular-se a maneiras prejudiciais de lidar com a doença - culpa, sensação de punição e abandono por Deus - que interferem na utilização adequada de recursos. Há casos, ainda, nos quais se observa que, para o doente, o acometimento pela enfermidade não condiz com suas atitudes e comportamentos, o que pode deflagrar questionamentos da própria fé permeados por sentimentos de raiva e revolta (Dalgalarrondo, 2008; Geertz, 1973/2008; Pargament, 1997; Pargament et al., 2000).

Cabe lembrar que, para algumas religiões, a punição não é percebida de modo negativo, pois a ela se atribuem meios de evitar ou eliminar comportamentos julgados incompatíveis no contexto de uma determinada crença. Também é possível que o sofrimento seja considerado uma expressão do percurso espiritual de alguém (como no espiritismo). Assim, 
em razão da diversidade de crenças e dos impactos gerados pelos sistemas religiosos, é inconcebível qualificar a religião como algo "bom" ou "ruim","funcional" ou "disfuncional", devendo-se avaliar cada caso conforme suas particularidades (Geertz, 1973/2008; James, 1902/1994).

A preparação do profissional da saúde sobre a temática religiosa/ espiritual foi discutida pelos integrantes de ambos os grupos.

... acho que esse é um tema que deveria entrar na formação, pois ampliaria a visão dos profissionais no sentido do respeito, inclusive, da diversidade religiosa, e de como lidar com os momentos em que há discordâncias que interferem no tratamento. (P3)

Não. Nunca estou preparada. (N2)

... a resistência também acontece por falta de conhecimento mesmo. Como não se sabe abordar... se afastam da questão (P1)

Já tive que recusar realizar uma cirurgia devido ao elevado risco já que a paciente se negava a receber transfusão... (M1)

Os integrantes dos grupos foram, de maneira geral, favoráveis à criação de disciplinas que preparem o profissional sobre a temática religiosa/espiritual. No Brasil, são escassos os cursos que abarquem em seus currículos conteúdos sobre a interface religião e/ou espiritualidade e a área da saúde, reservando-se o assunto para momentos práticos de ensino (Dal-Farra \& Geremia, 2010). Todavia, nos Estados Unidos cerca de $90 \%$ dos cursos de medicina possuem disciplinas que direta ou indiretamente abordam espiritualidade e saúde incluindo tópicos sobre as consequências da religiosidade e espiritualidade para a saúde, maneiras de se levantar a história espiritual do paciente, aspectos éticos e possíveis impactos para as decisões médicas (Koenig et al., 2010).

A falta de treinamento e de habilidade em identificar as demandas dos pacientes impelem a negação ou a rejeição da dimensão espiritual (McCauley et al., 2005; Monroe et al., 2003). Ambas as nutricionistas relataram não se sentirem capacitadas para lidar com a temática religiosa/espiritual, realçando a tradição biomédica da categoria. Estudos brasileiros apontam a percepção de despreparo de docentes e futuros enfermeiros (Peixoto, 2006; Tomasso et al., 2011), revelando que essa dificuldade atinge também a área da Enfermagem.

Estudiosos sugerem que o aspecto cultural está relacionado ao modo como as pessoas vivenciam a relação entre saúde e religiosidade/ espiritualidade (Balboni et al., 2007; Koenig et al., 2004). Tendo em vista que o Brasil é caracterizado pelo pluralismo religioso e pela complexidade cultural, cuja população atribui grande importância à religião (Dal-Farra \& Geremia, 2010; Dalgalarrondo, 2008; Moreira-Almeida et al., 2010), os pro- 
fissionais de saúde necessitam de preparo para lidar com essa temática em seus atendimentos.

No que se refere a crenças, foram debatidas questões sobre a necessidade de respeitar os pacientes, além da possibilidade de ocorrer conflitos quando as crenças de profissionais e pacientes/familiares são divergentes.

O que me parece ser um ponto significativo é que a 'minha'religião/religiosidade às vezes dificulta uma discussão mais ampla. (P2)

.... mesmo que o profissional cuidador não acredite, ele precisa respeitar a crença do paciente. (N2)

Penso que esse cuidado deve começar por cada profissional se apropriar de suas concepções, religiosas, existenciais; para saber de que forma lidar com as diferenças no contato com os pacientes ( $\mathrm{P} 1$ )

Por se tratar de uma doença potencialmente fatal, o câncer remete à ideia de finitude, suscitando reflexões sobre o sentido da vida e da morte tanto para os pacientes como para os profissionais que atuam na assistência oncológica. Conforme o relato de P1, é desejável que os profissionais de saúde reflitam sobre suas próprias crenças, evitando interferências negativas nos cuidados do paciente (Ecklund et al., 2007; Peixoto, 2006). Tendo em vista tais preocupações, sublinha-se a importância da atuação do psicólogo na equipe visando auxiliá-los a identificar e pensar sobre suas crenças (Espíndula et al., 2010; Junqueira, 2008).

Idealmente, a postura do profissional deveria promover um ambiente acolhedor e suportivo ao paciente, mesmo se houver divergências entre suas crenças religiosas e o tratamento. De acordo com alguns autores, os profissionais podem, inclusive, introduzir assuntos sobre religiosidade e espiritualidade nos atendimentos clínicos desde que sejam respeitados os preceitos seguidos pelos pacientes (Koenig, 2006; Panzini \& Bandeira, 2007; Saad \& Battistella, 2001).

Os relatos a seguir se referem à assistência religiosa no contexto oncológico, bem como à relação entre profissionais da saúde e representantes religiosos.

É comum que os representantes religiosos visitem os pacientes hospitalizados para fazer orações. (P2)

Eu não tenho um diálogo sistemático... os contatos em geral são feitos mediante necessidades específicas dos pacientes que atendo... mas acho que deveria ter mais diálogo. (P3) 
Em suma, é importante reconhecer que, em momentos de sofrimento, os pacientes oncológicos podem recorrer à fé e às crenças religiosas como fonte de alívio e conforto (Aquino \& Zago, 2007; Barbosa \& Freitas, 2009; Fitchett \& Canada, 2010; Thuné-Boyle et al., 2011). Faz-se premente, portanto, a criação de serviços de suporte religioso/espiritual nos hospitais brasileiros de forma a atender as demandas dos pacientes. Segundo Fitchett et al. (2010), o encaminhamento aos capelães é indicado para situações em que o paciente/familiar se encontra longe de sua comunidade religiosa, quando membros da igreja não se sentem confortáveis ou não têm tempo para frequentar o hospital.É possível, ainda, que os capelães atendam às necessidades espirituais dos pacientes não obrigatoriamente vinculadas a uma doutrina religiosa.

\section{Considerações finais}

Na presente pesquisa, predominou a percepção sobre a influência positiva da religiosidade e espiritualidade para a saúde, sendo os efeitos negativos identificados em menor proporção.

Constatou-se, também, a escassez de serviços de apoio religioso nos hospitais brasileiros, o que confirma a necessidade de um trabalho conjunto entre profissionais da saúde e representantes religiosos vinculados ou não às instituições hospitalares, em prol de um atendimento integral para o paciente oncológico. Além disso, o aprimoramento da prática profissional requer capacitação e treinamento adequados para a abordagem da temática religiosa/espiritual nos atendimentos.

Éigualmente interessante destacar algumas vantagense desvantagens quanto ao uso de ferramentas eletrônicas para coleta de dados em pesquisas (Flick, 2009). Por um lado, elimina-se a transcrição de informações para constituição de um banco de dados, assegura-se maior liberdade de expressão sobre temas controversos e amplia-se a participação e interação de participantes de regiões geograficamente distantes. Mas, por outro lado, exige-se acesso e familiaridade com tais recursos (por exemplo, a internet) e inviabilizam-se esclarecimentos em tempo real (no caso, do preenchimento do questionário eletrônico). Recomenda-se a colaboração de auxiliares de pesquisa para otimizar a coleta e superar as dificuldades operacionais.

Sugerem-se pesquisas futuras que contemplem a abordagem da temática nos atendimentos de maneira mais aprofundada com o intuito de instrumentalizar o profissional para a sua prática. A opinião de categorias profissionais não representadas neste estudo, como a Enfermagem, também deve ser considerada. Ademais, a religiosidade/espiritualidade dos próprios profissionais da saúde pode ser focalizada, incluindo outros instrumentos que avaliem qualidade de vida e bem-estar espiritual. 


\section{Religiosity and spirituality in oncology: Health Professionals' conceptions}

Abstract: Specialized literature has been identifying positive and negative influences of religious and spiritual beliefs in coping with diseases such as cancer. Therefore, this research aimed to identify, analyze and understand the concepts of health professionals in regards to the association amongst religiosity, spirituality and health in Oncology. In an initial stage, an electronic questionnaire was answered by 85 professionals. In the later stage, seven professionals participated in online focus groups. Results indicated that approximately half of the participants of the first stage do not practice religious activities. However, $85 \%$ revealed a high degree of spirituality. Religious/spiritual themes are common in clinical setting. Group members reported not having received academic training to address such issues. Nonetheless, participants showed positive views as to the influence of religiosity/spirituality on health, with negative effects to a lesser extent. Further studies may support programs aimed at training future professionals in the field of oncology.

Keywords: Religiosity. Spirituality. Coping. Cancer. Health professionals.

\section{Religiosité, spiritualité en oncologie: concepts de professionnels de la santé}

Résumé:Plusieurs études soulèvent des influences positives et négatives des croyances religieuses et spirituelles pour faire face à des maladies comme le cancer. Ainsi, cette recherche vise à comprendre et analyser les idées de professionnels de la santé sur l sassociation religiosité, spiritualité et santé, en Oncologie. Dans un premier temps, on a utilisé un questionnaire électronique, qui a été répondu par 85 professionnels. Ensuite, sept professionnels ont participé aux groupes de discussion en ligne. À peu prés la moitié de l'échantillon n'a pas des activités religieuses. Toutefois, $85 \%$ a montré un haut degré de spiritualité. Thèmes religieux/spirituel sont fréquents dans les consultations. Les membres des groupes ont déclaré ne pas avoir de formation pour traiter des questions semblables. En somme, les professionnels ont une opinion positive sur la religiosité, mais reconnaissent aussi ses effets indésirables. Des recherches sont encore nécessaires pour mieux baser la formation du personnel en Oncologie.

Mots-clés: Religiosité. Spiritualité. Coping. Cancer. Professionnels de la santé.

\section{Religiosidad y espiritualidad en oncología: Concepciones de profesionales de la salud}

Resumen: La literatura especializada viene identificando influencias positivas y negativas de creencias religiosas y espirituales en el enfrentamiento de enfermedades, como el cáncer. La presente investigación objetivó conocer, analizar 
y comprender las concepciones de profesionales de salud respecto a la asociación entre religiosidad, espiritualidad y salud en Oncología. Primeramente, se utilizó un cuestionário electrónico que fue contestado por 85 profesionales. Posteriormente, siete profesionales participaron de grupos focales online. Los resultados indicaron que aproximadamente la mitad de los participantes de la primera etapa no practican actividades religiosas. Todavía, $85 \%$ revelaron alto grado de espiritualidad. Temas religiosos/espirituales son frecuentes en los atendimientos. Los integrantes del los grupos relataron que no recibieron formación academica para abordar la temática. En suma, los participantes evidenciaron concepciones positivas sobre la influencia de la religiosidad/espiritualidad a la salud, con efectos negativos en menor proporción. Futuras investigaciones pueden apoyar programas de capacitación de futuros profesionales en la Oncología.

Palabras clave: Religiosidad. Espiritualidad. Enfrentamiento. Cáncer. Profesionales de la salud.

\section{Referências}

Alves, V. P. (2004). Fenomenologia da religião: pesquisas sobre a experiência religiosa com universitários e suas implicações para o ensino religioso. In A. Holanda (Org.), Psicologia, religiosidade e fenomenologia (pp. 79-95). Campinas, SP: Alínea.

Aquino, V. V., \& Zago, M. M. F. (2007). O significado das crenças religiosas para um grupo de pacientes oncológicos em reabilitação. Revista Latino-Americana de Enfermagem, 15(1), 42-47.

Balboni, T. A., Vanderwerker, L. C., Block, S. D., Paulk, M. E., Lathan, C. S., Peteet, J. R., \& Prigerson, H. G. (2007). Religiousness and spiritual support among advanced cancer patients and associations with end-of-life treatment preferences and quality of life. Journal of Clinical Oncology, 25(5), 555-560.

Barbosa, K. A., \& Freitas, M. H. (2009). Religiosidade e atitude diante da morte em idosos sob cuidados paliativos. Revista Kairós, 12(1), 113-134.

Bardin, L. (2009). Análise de conteúdo (5a ed.). Lisboa, Portugal: Edições 70.

Castañon, G. (2007). Introdução à Epistemologia. São Paulo, SP: EPU.

Constituição da República Federativa do Brasil de 1988. (1998). Brasília, DF. Recuperado de http://www.planalto.gov.br 
Dal-Farra, R. A., \& Geremia, C. (2010). Educação em saúde e espiritualidade: proposições metodológicas. Revista Brasileira de Educação Médica, 34(4), 587-597.

Dalgalarrondo, P. (2008). Religião, psicopatologia e saúde mental. Porto Alegre, RS: Artmed.

Decreto n. 30.582, de 16 de julho de 2009. (2009). Regulamenta a prestação de assistência religiosa nas entidades civis e militares de internação coletiva de que trata a Lei n³.216, de 5 de novembro de 2003, alterada pela Lei n 3.540, de 11 de janeiro de 2005, no âmbito do Distrito Federal. Brasília, DF. Recuperado de http://www.cl.df.gov.br

Ecklund, E. H., Cadge, W., Gage, E. A., \& Catlin, E. A. (2007). The religious and spiritual beliefs and practices of academic pediatric oncologists in the United States. Journal of Pediatric Hematology/Oncology, 29(11), 736-742.

Espíndula, J. A., Valle, E. R. M., \& Bello, A. A. (2010). Religião e espiritualidade: um olhar de profissionais de saúde. Revista Latino-Americana de Enfermagem, 18(6). Recuperado de http://www.scielo.br

Faria, J. B. (2004). Religiosidade, enfrentamento e bem-estar subjetivo em pessoas vivendo com HIV/AIDS (Dissertação de Mestrado). Universidade de Brasília, Brasília, DF.

Faria, J. B., \& Seidl, E. M. F. (2005). Religiosidade e enfrentamento em contextos de saúde e doença. Psicologia: Reflexão e Crítica, 18(3), 381-389.

Fetzer Institute, National Institute on Aging Working Group. (1999). Multidimensional measurement of religiousness/spirituality for use in health research: $A$ report of a national working group. Fetzer Institute, Kalamazoo, MI. Recuperado de http:// www.fetzer.org/research/248-dses

Fitchett, G., \& Canada, A. L. (2010). The hole of religion/spirituality in coping with cancer: evidence, assessment, and intervention. In J. C. Holland, W. S. Breitbart, P. B. Jacobsen, M. S. Lederberg, M. J. Loscalzo \& R. McCorkle (Ogs.), Psycho-oncology (pp. 440-446). New York, NY: Oxford University Press.

Fitchett, G., King, S. D. W., \& Vandenhoeck, A. (2010). Education of chaplains in psycho-oncology. In J. C. Holland, W. S. Breitbart, P. B. Jacobsen, M. S. Lederberg, M. J. Loscalzo \& R. McCorkle (Orgs.), Psycho-oncology (pp. 605-609). New York, NY: Oxford University Press.

Flick, U. (2009). Pesquisa qualitativa online: a utilização da internet. In U. Flick (Ed.), Introdução à pesquisa qualitativa (pp. 238-253). Porto Alegre, RS: Artmed. 
Folkman, S., Lazarus, R. S., Gruen, R. J., \& DeLongis, A. (1986). Appraisal, coping, health status, and psychological symptoms. Journal of Personality and Social Psychology, 50(3), 571-579.

Geertz, C. (2008). A religião como sistema cultural. In C. Geertz, A interpretação das culturas (pp. 65-91). Rio de Janeiro, RJ: Livros Técnicos e Científicos (Trabalho original publicado em 1973)

Gentil, R. C., Guia, B. P., \& Sanna, M. C. (2011). Organização serviços de capelania hospitalar: estudo bibliométrico. Escola Anna Nery Revista de Enfermagem, 15(1), 162-170.

Gurung, R. A. R. (2010). Health Psychology: A cultural approach (2a ed.). Wadsworth, $\mathrm{OH}$ : Cengage Learning.

Hoffmann, F. S, Müller, M. C., \& Frasson, A. L. (2006). Repercussões psicossociais, apoio social e bem-estar espiritual em mulheres com câncer de mama. Psicologia, Saúde \& Doenças, 7(2), 239-254.

Hoffmann, F. S, Müller, M. C., \& Rubin, R. (2006). A mulher com câncer de mama: apoio social e espiritualidade. Mudanças: Psicologia da Saúde, 14(2), 143-150.

James, W. (1994). The varieties of religious experience. New York, NY: The Modern Library. (Trabalho original publicado em 1902)

Junqueira, L. C.U. (2008). Vivências de médicos oncologistas: um estudo da religiosidade no cuidado existencial em saúde. (Dissertação de mestrado), Universidade de São Paulo, Ribeirão Preto, SP.

Koenig, H. G. (2006). The spiritual history. Southern Medical Journal, 99(10), 1159-1160.

Koenig, H. G. (2009). Research on religion, spirituality, and mental health: A review. Canadian Journal of Psychiatry, 54(5), 283-291.

Koenig, H. G., George, L. K., Titus, P., \& Meador, K. G. (2004). Religion, spirituality, and acute care hospitalization and long-term care use by older patients. Archives of Internal Medicine, 16, 1579-1585.

Koenig, H. G., Hooten, E. G., Lindsay-Calkins, E., \& Meador, K. G. (2010). Spirituality in medical school curricula: Findings from a national survey. International Journal of Psychiatry in Medicine, 40(4), 391-398.

Lei n. 9.982, de 14 de julho de 2000. (2000). Dispõe sobre a prestação de assistência religiosa nas entidades hospitalares públicas e privadas, bem como nos 
estabelecimentos prisionais civis e militares. Brasília, DF. Recuperado de http:// www.planalto.gov.br

Liberato, R. P., \& Macieira, R. C. (2008). Espiritualidade no enfrentamento do câncer. In V. Carvalho, M. H. P. Franco, M. J. Kovács, R. P. Liberato, R. C. Macieira, M. T. Veit, M. J. B. Gomes \& L. H. C. Barros (Orgs.), Temas em psico-oncologia (pp. 414-431). São Paulo, SP: Summus.

Lucchetti, G., Almeida, L. G. C., \& Granero, A. L. (2010). Espiritualidade no paciente em diálise: o nefrologista deve abordar? Jornal Brasileiro de Nefrologia, 32(1), 128-132.

Maciejewski, P. K., Phelps, A. C., Kacel, E. L., Balboni, T. A., Balboni, M., Wright, A., ... Prigerson, H. G. (2012). Religious coping and behavioral disengagement: Opposing influences on advance care planning and receipt of intensive care near death. Psycho-Oncology, 21(7), 714-723. doi 10.1002/pon.1967

McCauley, J., Jenckes, M. W., Tarpley, M. J., Koenig, H. G., Yanek L. R., \& Becker, D. M. (2005). Spiritual beliefs and barriers among managed care practitioners. Journal of Religion and Health, 44(2), 137-146.

Monroe, M. H., Bynum, D., Susi, B., Phifer, N., Schultz, L., Franco, M.,... Garret, J. (2003). Primary care physician preferences regarding spiritual behavior in medical practice. Archives of Internal Medicine, 163(22), 2751-2756. doi:10.1001/ archinte.163.22.2751

Moreira-Almeida, A. (2010). O crescente impacto das publicações em espiritualidade e saúde e o papel da Revista de Psiquiatria Clínica. Revista de Psiquiatria Clínica, $37(2), 41-42$.

Moreira-Almeida, A., Pinsky, I., Zaleski, M., \& Laranjeira, R. (2010). Envolvimento religioso e fatores sociodemográficos: resultados de um levantamento nacional no Brasil. Revista de Psiquiatria Clínica, 37(1), 12-15.

Oliveira, M. S., Fernandes, A. F. C., \& Galvão, M. T. G. (2005). Mulheres vivenciando o adoecer em face do câncer cérvico-uterino. Acta Paulista de Enfermagem, 18(2), 150-155.

Organização Mundial da Saúde. (1999). Amendments to the Constitution. Recuperado de http://www.who.int/en/

Paiva, G. J. (2002). Ciência, religião, psicologia: conhecimento e comportamento. Psicologia: Reflexão e Crítica, 15(3), 561-567. 
Paiva, G. J., Zangari, W., Verdade, M. V., Paula, J. R. M., Faria, D. G. R., Gomes, D. M., ... Gomes, A. M. A. (2009). Psicologia da religião no Brasil: a produção em periódicos e livros. Psicologia: Teoria e Pesquisa, 25(3), 441-446.

Panzini, R. G. (2004). Escala de coping religioso-espiritual (Escala CRE): tradução, adaptação e validação da escala RCOPE, abordando relações com saúde e qualidade de vida (Dissertação de Mestrado). Universidade Federal do Rio Grande do Sul. Porto Alegre, RS.

Panzini, R. G., \& Bandeira, D. R. (2007). Coping (enfrentamento) religioso/espiritual. Revista de Psiquiatria Clínica, 34(1), 126-135.

Pargament, K. I. (1997). The psychology of religion and coping. New York, NY: The Guilford Press.

Pargament, K. I., Koenig, H. G., \& Perez, L. M. (2000). The many methods of religious coping: development and initial validation of the RCOPE. Journal of Clinical Psychology, 56(4), 519-543.

Pargament, K. I., Smith, B. W., Koenig, H. G., \& Perez, L. (1998). Patterns of positive and negative religious coping with major life stressors. Journal for the Scientific Study of Religion, 18, 710-724.

Pargament, K. I., Tarakeshwar, N., Ellison, C. G., \& Wulff, K. M. (2001). Religious coping among the religious: the relationships between religious coping and well-being in a national sample of Presbyterian clergy, elders and members. Journal for the Scientific Study of Religion, 30(3), 497-513.

Peixoto, L. F. (2006). A assistência psicoespiritual na Enfermagem: ensino e prática (Tese de Doutorado). Pontifícia Universidade Católica de São Paulo, São Paulo, SP.

Post, S. G., Puchalski, C. M., \& Larson, D. B. (2000). Physicians and patient spirituality: Professional boundaries, competency, and ethics. Annals of Internal Medicine, 132(7), 578-583.

Prada, A. A. (2006). Manual de psicooncología. Bogotá, Colombia: Javegraf.

Rocha, N. S., Panzini, R. G., Pargendler, J. S., \& Fleck, M. P. A. (2008). Desenvolvimento do módulo para avaliar espiritualidade, religiosidade e crenças pessoais do WHOQOL (WHOQOL-SRPB). In M. P. A. Fleck (Org.), A avaliação de qualidade de vida (pp. 93-101). Porto Alegre, RS: Artmed.

Saad, M., Masiero, D., \& Battistella, L. R. (2001). Espiritualidade baseada em evidências. Acta Fisiátrica 8(3), 107-112. 
Straub, R. O. (2005). Psicologia da saúde (R. C. Costa, trad.). Porto Alegre, RS: Artmed.

Sulmasy, D. P. (2009). Spirituality, religion, and clinical care. Chest, 135, 1634-1642.

Tarakeshwar, N., Vanderwerker, L. C., Paulk, E., Pearce, M. J., Kasl, S. V., \& Prigerson, H. G. (2006). Religious coping is associated with the quality of life of patients with advanced cancer. Journal of Palliative Medicine, 9(3), 646-657.

Teixeira, J. J. V., \& Lefèvre, F. (2008). Significado da intervenção médica e da fé religiosa para o paciente idoso com câncer. Ciência \& Saúde Coletiva, 13(4),1247-1256.

Thuné-Boyle, I. C. V., Stygall1, J., Keshtgar, M. R. S., Davidson, T. I., \& Newman, S. P. (2011). Religious coping strategies in patients diagnosed with breast cancer in the UK. Psycho-Oncology, 20(7), 771-782.

Tix, A. P., \& Frazier, P. A. (1998). The use of religious coping during stressful life events: Main effects, moderation, and mediation. Journal of Consulting and Clinical Psychology, 66(2), 411-422.

Tomasso, C. S., Beltrame, I. L., \& Lucchetti, G. (2011). Knowledge and attitudes of nursing professors and students concerning the interface between spirituality, religiosity and health. Revista Latino-Americana de Enfermagem, 19(5), 1205-1213.

Zinnbauer, B. J., \& Pargament, K. I. (2004). Religiousness and spirituality. In R. F. Paloutzian \& C. L. Park (Eds.), Handbook of the psychology of religion and spirituality (pp. 21-42). New York, NY: Guilford Press. 
Caroline Amado Gobatto, psicóloga, mestranda do Programa de Pós-Graduação Processos de Desenvolvimento Humano e Saúde da Universidade de Brasília. Residente Multiprofissional do Hospital Universitário de Brasília. Endereço para correspondência:CLN 411 Bloco E Sala 217, Asa Norte, Brasília, DF. CEP 70866-550. Endereço eletrônico: carolinegobatto@yahoo.com.br

Tereza Cristina Cavalcanti Ferreira de Araujo, Professora Associada da Universidade de Brasília, pesquisadora do Conselho Nacional de Desenvolvimento Científico e Tecnológico (CNPq), Coordenadora do Laboratório de Saúde e Desenvolvimento Humano, Pós-Doutora pela Unesco (Paris, França), Doutora pela Université de Paris$X$ Nanterre. Endereço para correspondência: Universidade de Brasília, Campus Darcy Ribeiro, Instituto de Psicologia, Asa Norte, Brasília, DF. CEP 70910-900. Endereço eletrônico:araujotc@unb.br

Recebido: $27 / 03 / 2012$

Aceito: $31 / 10 / 2012$ 